OPEN ACCESS

Edited by:

Jonathan Spencer West, Rothamsted Research,

United Kingdom

Reviewed by:

Kevin King,

Rothamsted Research,

United Kingdom

Marzia Beccaccioli,

Sapienza University of Rome, Italy

*Correspondence:

Annette Pford

annette.pfordt@uni-goettingen.de

Specialty section:

This article was submitted to

Disease Management,

a section of the journal

Frontiers in Agronomy

Received: 31 March 2020

Accepted: 28 August 2020

Published: 29 September 2020

Citation:

Pfordt A, Schiwek S, Karlovsky P and von Tiedemann A (2020) Trichoderma

Afroharzianum Ear Rot-A New

Disease on Maize in Europe.

Front. Agron. 2:547758

doi: 10.3389/fagro.2020.547758

\section{Trichoderma Afroharzianum Ear Rot-A New Disease on Maize in Europe}

\author{
Annette Pfordt ${ }^{1 *}$, Simon Schiwek ${ }^{2}$, Petr Karlovsky ${ }^{2}$ and Andreas von Tiedemann ${ }^{1}$ \\ ${ }^{1}$ Plant Pathology and Crop Protectison, University of Goettingen, Goettingen, Germany, ${ }^{2}$ Molecular Phytopathology and \\ Mycotoxin Research, University of Goettingen, Goettingen, Germany
}

Trichoderma species are widespread filamentous fungi in soils, on plant roots and decaying plant residues. Due to their strong competitiveness and mycoparasitic activity against other fungi, particular strains of Trichoderma sp. are used in agriculture as biocontrol agents against plant pathogens. Commercial products based on strains of T. harzianum or T. afroharzianum have been applied to control Rhizoctonia spp., Fusarium spp., and Phytophthora spp. in various crops. In 2018, however, severe infections of Trichoderma on maize ears were recorded for the first time in a field in Southern Germany. Infected maize cobs were sampled, the fungus was isolated in pure culture and preliminarily identified microscopically as T. harzianum. After silk channel inoculation in the greenhouse, ear rot disease of high severity was observed. In addition to fungal colonization, the dry matter content in cobs was significantly reduced compared to water inoculated cobs. In 2018 and 2019, a total of 13 T. harzianum isolates from maize cobs and maize stalks were isolated and tested, for pathogenicity on maize plants in the greenhouse, compared to several reference isolates. Four isolates proved to be highly aggressive, two biocontrol isolates, Trichodex (T39), and strain T12, induced slight infection and eleven isolates were non-pathogenic. After sequencing of the translation elongation factor- $1 \alpha$ (tef- $1 \alpha)$ and internal transcribes spacers (ITS), the four highly aggressive isolates were reassigned to $T$. afroharzianum, while the commercial biocontrol isolates Trichodex (T39), and T12, as well as the other non-pathogenic strains belonged to T. harzianum, T. atroviride, or T. tomentosum. This, to our knowledge, is the first report on Trichoderma sp. as a pathogen causing ear rot disease in maize in Europe with the potential to incite significant yield losses. We therefore propose to name this disease as "Trichoderma ear rot on maize".

Keywords: Trichoderma harzianum, Trichoderma afroharzianum, pathogenicity, Trichoderma ear rot on maize, maize

\section{INTRODUCTION}

Members of the genus Trichoderma are classified as imperfect fungi in the division Ascomycota and are ubiquitous in various types of soil. Some species of Trichoderma have biocontrol potential and can suppress pathogen growth by direct and indirect mechanisms including mycoparasitism, antibiosis, induction of host resistance and competition for nutrients and space (Kubicek et al., 2008; Jaklitsch and Voglmayr, 2015; Samuels and Hebbar, 2015; Ghazanfar et al., 2018). 
Especially, the ability to detoxify zearalenone protects Trichoderma spp. from the chemical defense of Fusarium spp. (Popiel et al., 2014). They can thereby control and antagonize a broad range of economically important plant parasitic pathogens (Ferrigo et al., 2014; Gupta et al., 2014; Harman, 2015). In addition, they may increase plant resilience against drought conditions and promote shoot and root growth (Arora et al., 2003). Harman et al. (2004b) reported a significant yield increase in maize due to Trichoderma treatments. Apart from the control of root and foliar pathogens, Trichoderma spp. enhance nutrient solubilization and uptake as well as root and root hair development (Herrera-Estrella and Chet, 2004; Schuster and Schmoll, 2010).

Trichoderma species have been described as opportunistic, basically avirulent plant symbionts in soil (Harman et al., 2004a); however, a few reports have mentioned Trichoderma as ear rot pathogen on maize in the US (Munkvold and White, 2016; Wise et al., 2016; OSU., 2020). Trichoderma ear rot infection has been characterized by the occurrence of dark, blue-green layers of conidia on and between the kernels of infected ears causing premature germination of the kernels (Wise et al., 2016). In addition, the dry matter content of ears infected with Trichoderma was strongly reduced compared to uninfected ears. The occurrence of Trichoderma ear rot was associated with injuries caused by feeding birds or other mechanical damage in Kentucky and Ohio (Vincelli, 2014).

Surprisingly, in 2018 a severe occurrence of Trichoderma on the maize cobs was recorded at a field site in Southern Germany. Cobs sampled from 20 maize varieties were overgrown with mycelium producing green layers of conidia between the kernels and on the outside of the husk leaves. Similar disease symptoms have been previously observed in Southern Bavaria, after warm, and dry summers.

The aim of the present study was to identify and verify Trichoderma as a new pathogen causing ear rot disease on maize. Therefore, Trichoderma-infected cobs from four locations in Germany and France were sampled, cultured, and microscopically examined as well as analyzed by sequencing the gene for translation elongation factor- $1 \alpha($ tef- $1 \alpha)$ and internal transcribes spacers (ITS). Furthermore, pathogenicity of Trichoderma isolates and the impact of infection on dry matter content of maize cobs were tested after artificial inoculation at flowering in the greenhouse.

\section{MATERIALS AND METHODS}

\section{Fungal Isolation and Cultivation}

Maize cobs and stalks were collected from naturally infected silage and grain maize in Germany in 2018 and 2019. Thirty randomly chosen kernels from each cob were surface sterilized for $10 \mathrm{~min}$ with $0.1 \%$ silver nitrate and placed on potato dextrose agar (PDA). The stalk samples were cut in nine slices, surface sterilized as described above and placed on PDA plates. After 2 days, mycelium outgrown from the sample was transferred to PDA plates. Single conidia cultures were produced, and isolates were stored on synthetic low nutrition agar (SNA) plates at $4^{\circ} \mathrm{C}$.

\section{Inoculation Procedure}

Spores from single spore cultures were transferred to PDA plates containing antibiotics $(400 \mu \mathrm{g} / \mathrm{ml}$ streptomycin, Duchefa Biochemie, Haarlem, The Netherlands; $30 \mu \mathrm{g} / \mathrm{ml}$ rifampicin, AppliChem, Darmstadt, Germany) and incubated under NearUV-light $(\lambda=340-400 \mathrm{~nm})$ at $23^{\circ} \mathrm{C}$ in a growth chamber. After 2 weeks, sterile water was added to plates and conidia were scraped off with a microscope slide. The conidia suspension was then filtered through gauze and cell density was measured with a Thoma haemocytometer and adjusted to $1 \times 10^{6}$ conidia per ml. Primary ears of maize plants were inoculated seven days after silk channel emergence (BBCH 65). For this purpose, $1 \mathrm{ml}$ of conidia suspension was injected with a syringe (Braun, Melsungen, Germany) into the silk channel between the cob tip and the point where silks emerge from the husk.

\section{Plant Cultivation and Pathogenicity Assessment on Maize Ears}

Maize seeds of two varieties were sown in a mixture of soil (potting soil/compost/sand mixture of $1: 2: 1$ ) in $20 \mathrm{~cm}$ diameter pots. Pots were placed in the greenhouse at $23^{\circ} \mathrm{C}$ under a seasonal day-/night light cycle. Five plants per isolate were inoculated by silk channel injection and five additional plants were inoculated with water, as control. Thirteen Trichoderma isolates originally isolated from maize cobs in the field were compared to four reference strains of T. harzianum (IPP0318, IPP0319, IPP0320, T12), one type strain of T. afroharzianum (CBS 124620) and one strain of T. atroviride (IPP0316) obtained from different fungal collections (see Table 1). In addition, one commercial biocontrol isolate, T39 (Trichodex), and one isolate, T12, with potential biocontrol activity (kindly provided by the Department for Phytopathology of Justus-Liebig-University, Gießen, Germany) were tested. Four weeks ( $28 \mathrm{dpi}$ ) after inoculation, husk leaves of inoculated and control ears were removed, and disease severity was assessed visually according to the EPPO guidelines (European Mediterranean Plant Protection Organization., 2015) as the percentage $(0-100 \%)$ of the cob surface covered with fungal mycelium. Finally, cobs were weighed and dried for 5 days at $60^{\circ} \mathrm{C}$ to assess the dry matter content. In addition, isolates were re-isolated and cultured on PDA to confirm the Koch's Postulate.

\section{DNA Extraction and Phylogenetic Analysis}

Total DNA was extracted from lyophilized mycelium of single spore cultures by using a CTAB-based method described by Brandfass and Karlovsky (2008). Quality and quantity of the extracted DNA was assessed by agarose gel electrophoresis $(60 \mathrm{~min}$ at $4.6 \mathrm{~V} / \mathrm{cm})$ and stained with ethidium bromide. Partial translation elongation factor- $1 \alpha$ $(t e f-1 \alpha)$ and internal transcribed spacer (ITS) were used to differentiate within the Trichoderma harzianum complex. Amplification was performed in a peqSTAR96 universal gradient thermocycler (PEQLAB, Erlangen, Germany) using 1:100 dilution of DNA extract in a total reaction volume of $25 \mu \mathrm{l}$. Marker genes tef- $1 \alpha$ and ITS were amplified with the primers EF1 (ATGGGTAAGGARGACAAGAC) and EF2 (GGARGTACCAGTSATCATGTT) (O’Donnell et al., 
TABLE 1 | Host, geographic locations, and year of isolation of Trichoderma isolates used in this study.

\begin{tabular}{|c|c|c|c|c|}
\hline Isolates & Species & Year & Location & Source \\
\hline Tri1 & Trichoderma afroharzianum & 2018 & Croix de Pardies (F) & Zea mays (cob)! \\
\hline Tri2 & Trichoderma afroharzianum & 2018 & Kuenzing (D) & Z. mays (cob)! \\
\hline Tri3 & Trichoderma afroharzianum & 2018 & Pocking (D) & Z. mays (cob)! \\
\hline Tri4 & Trichoderma tomentosum & 2018 & Altoetting (D) & Z. mays (cob)! \\
\hline Tri5 & Trichoderma afroharzianum & 2019 & Bernburg (D) & Z. mays (cob)! \\
\hline IPP0316 & Trichoderma atroviride & 1976 & - & Baby food \\
\hline IPP0318 & Trichoderma harzianum & 1992 & Mae Hia (T) & Soil \\
\hline IPP0319 & Trichoderma harzianum & 1992 & Chiang Mai $(T)$ & Soil \\
\hline IPP0320 & Trichoderma harzianum & 1992 & Mae Hia (T) & Soil \\
\hline Tri6 & Trichoderma harzianum & 2019 & Kleinwanzleben (D) & Z. mays (cob) \\
\hline Tri7 & Trichoderma harzianum & 2019 & Grucking (D) & Z. mays (cob) \\
\hline Tri8 & Trichoderma harzianum & 2019 & Loeningen (D) & Z. mays (cob) \\
\hline Tri9 & Trichoderma harzianum & 2019 & Loeningen (D) & Z. mays (cob) \\
\hline Tri10 & Trichoderma harzianum & 2019 & Großumstadt (D) & Z. mays (cob) \\
\hline Tri11 & Trichoderma harzianum & 2019 & Pfaffenhofen (D) & Z. mays (cob) \\
\hline Tri12 & Trichoderma harzianum & 2019 & Pfaffenhofen (D) & Z. mays (stalk) \\
\hline Tri14 & Trichoderma harzianum & 2019 & Pfaffenhofen (D) & Z. mays (stalk) \\
\hline $\mathrm{T} 12^{*}$ & Trichoderma harzianum & - & - & - \\
\hline T39 (Trichodex) & Trichoderma harzianum & - & Tel Aviv (ISR) & - \\
\hline CBS 124620 & Trichoderma afroharzianum & - & Peru & T. cacao \\
\hline
\end{tabular}

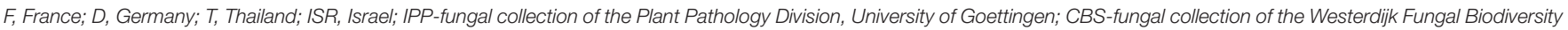

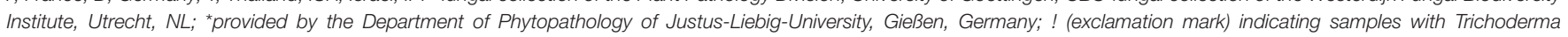
disease symptoms.

1998) and ITS1 (CTTGGTCATTTAGAGGAAGTAA) and ITS4 (TCCTCCGCTTATTGATATGC) (White et al., 1990), respectively. Reactions were carried out in a mixture of standard Taq reaction buffer $(10 \mathrm{mM}$ Tris- $\mathrm{HCl}, 50 \mathrm{mM} \mathrm{KCl}$, $1.5 \mathrm{mM} \mathrm{MgCl}_{2}, \mathrm{pH} 8.3$ at $25^{\circ} \mathrm{C}$; NEB), $100 \mu \mathrm{M}$ of each deoxyribonucleoside triphosphate, $0.3 \mu \mathrm{M}$ of each primer, $0.62 \mathrm{U}$ HotStart-polymerase (New England Biolabs), and $1 \mu \mathrm{L}$ template DNA solution. The final $\mathrm{MgCl}_{2}$ concentration was adjusted to $2 \mathrm{mM}$. The PCR cycling conditions for amplification of tef- $1 \alpha$ included an initial denaturation for $30 \mathrm{~s}$ at $95^{\circ} \mathrm{C}$; 30 cycles consisting of $30 \mathrm{~s}$ at $94^{\circ} \mathrm{C}, 30 \mathrm{~s}$ at $58^{\circ} \mathrm{C}$, and $1 \mathrm{~min}$ at $68^{\circ} \mathrm{C}$; and final extension for $5 \mathrm{~min}$ at $68^{\circ} \mathrm{C}$. The PCR cycling conditions for amplification of ITS included an initial denaturation for $30 \mathrm{~s}$ at $95^{\circ} \mathrm{C} ; 10$ cycles consisting of $30 \mathrm{~s}$ at $94^{\circ} \mathrm{C}$, a gradual decrease from $62^{\circ} \mathrm{C}$ to $53^{\circ} \mathrm{C}\left(-1^{\circ} \mathrm{C} /\right.$ cycle $)$ for $40 \mathrm{~s}$, and $1 \mathrm{~min}$ at $68^{\circ} \mathrm{C}$; 30 cycles of $30 \mathrm{~s}$ at $94^{\circ} \mathrm{C}, 40 \mathrm{~s}$ at $56^{\circ} \mathrm{C}$, and $1 \mathrm{~min}$ at $68^{\circ} \mathrm{C}$; and final extension for $5 \mathrm{~min}$ at $68^{\circ} \mathrm{C}$. Species were identified by multiple alignment of each sequence with reference sequences using ClustalW (Thompson et al., 1994) in MEGA Version 7.0.2 (Kumar et al., 2016).

\section{Statistical Analysis}

Statistical analysis was conducted with STATISTICA version 13 (Statistica GmbH, Germany). Differences between means of disease severity was analyzed using the non-parametric Kruskal-Wallis ANOVA by $5 \%$ probability. Analysis of variance (ANOVA) for dry matter content was carried out, followed by Tukey's-HSD-test at the $5 \%$ probability level.

\section{RESULTS}

\section{Geographic Origin of Samples}

In 2018, four isolates from four locations (Altoetting, Pocking, Kuenzing and Croix de Pardies) were obtained from maize cobs in Germany and France and subjected to further identification. In 2019, ten cobs from six locations, mainly from southern Germany, especially Bavaria, and along the Rhine valley (Bernburg, Kleinwanzleben, Grucking, Loeningen, Großumstadt, and Pfaffenhofen), were examined. In addition, two isolates from maize stalks from a single location (Pfaffenhofen) were obtained. Isolates Tri1, Tri2, Tri3, and Tri5 were isolated from maize cobs displaying strong Trichoderma infection, whereas the other Trichoderma isolates were obtained from cobs or stalks that did not induce any visual disease symptoms.

\section{Species Identification}

A molecular phylogenetic analysis was performed with thirteen Trichoderma isolates from maize cobs and six isolates obtained from fungal culture collections, based on partial tefl $\alpha$ (Figure 1) and ITS (Supplementary Figure 1). Sequences of 17 species in the T. harzianum complex (Chaverri et al., 2015) were selected from GenBank (Clark et al., 2015). Multiple gene alignments assigned eight isolates (Tri6-Tri12, Tri14) to T. harzianum, four isolates (Tri1-Tri3, Tri5) to the clade T. afroharzianum, and one isolate to T. tomentosum. Strains IPP0318-0320 were verified as T. harzianum and IPP0316 as T. atroviride. Isolates designated as T. harzianum clustered in two separate groups highly supported 


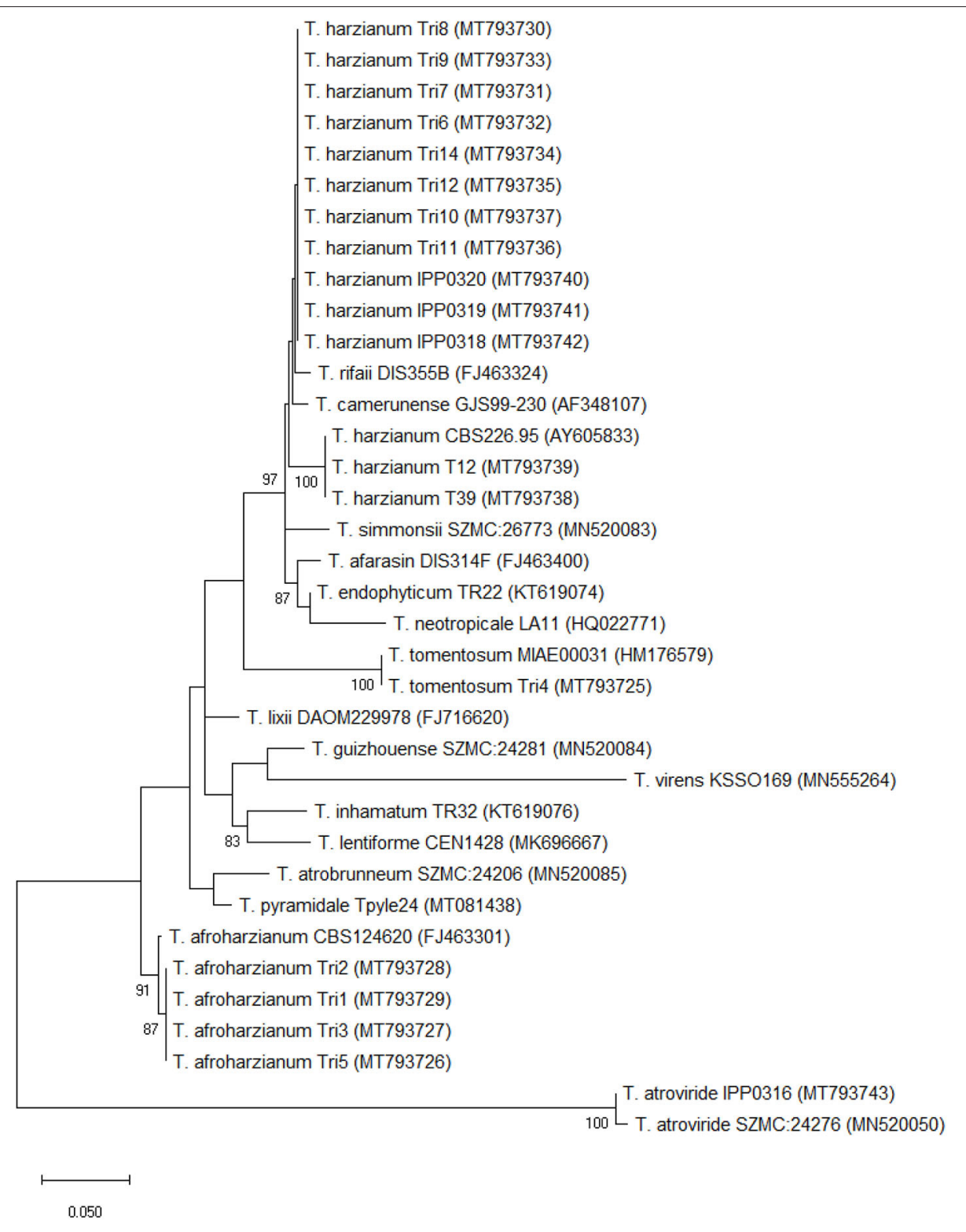

FIGURE 1 | Molecular phylogenetic analysis of translation elongation factor 1 alpha (tef- $1 \alpha$ ) by maximum likelihood method (1,000 bootstrap replicates) (Tamura et al., 2013). The analysis was performed with MEGA version 7.0.26 (Kumar et al., 2016) with partial tef- $1 \alpha$ sequences. The strains are listed in Table 1; GeneBank accession numbers of the sequences are shown in brackets. The tree is drawn to scale, with branch lengths measured in the number of substitutions per site. Bootstrap values are presented next to the nodes. Individual accession numbers are presented in Supplementary Table 1. Sequence alignment is available at TreeBASE (http://purl.org/phylo/treebase/phylows/study/TB2:S26786).

by bootstrap values. This result was largely confirmed by analysis of ITS sequences. Isolates Tri6-Tri12 an Tri14 showed high similarity to the selected references of T. harzianum, however, two isolates (T12 and T39) formed a separate clade, together with the reference sequence of T. harzianum CBS 226.95 (AY605833). All obtained sequences of $T$. afroharzianum clustered in one phylogenetic group highly supported by bootstrapping and showed $99.4 \%$ (523 out of 526) nucleotide similarity to the tef- $1 \alpha$ sequence of T. afroharzianum CBS124620 (FJ463301). Newly obtained sequences were deposited at Genbank under the accession numbers MT793725 to MT793743 for tef- $1 \alpha$ and
MT793744 to MT793762 for ITS, and sequence alignments were lodged at TreeBASE (http://purl.org/phylo/treebase/phylows/ study/TB2:S26786).

\section{Disease Symptoms and Severity on Maize Ears}

Trichoderma strains obtained from maize cobs displayed typical characteristics of this genus on PDA plates, such as initial growth of white mycelium, soon turning into green, and gray-green colonies, while the reverse side of the culture plates stayed uncoloured or light yellow. Trichoderma ear rot infection is 

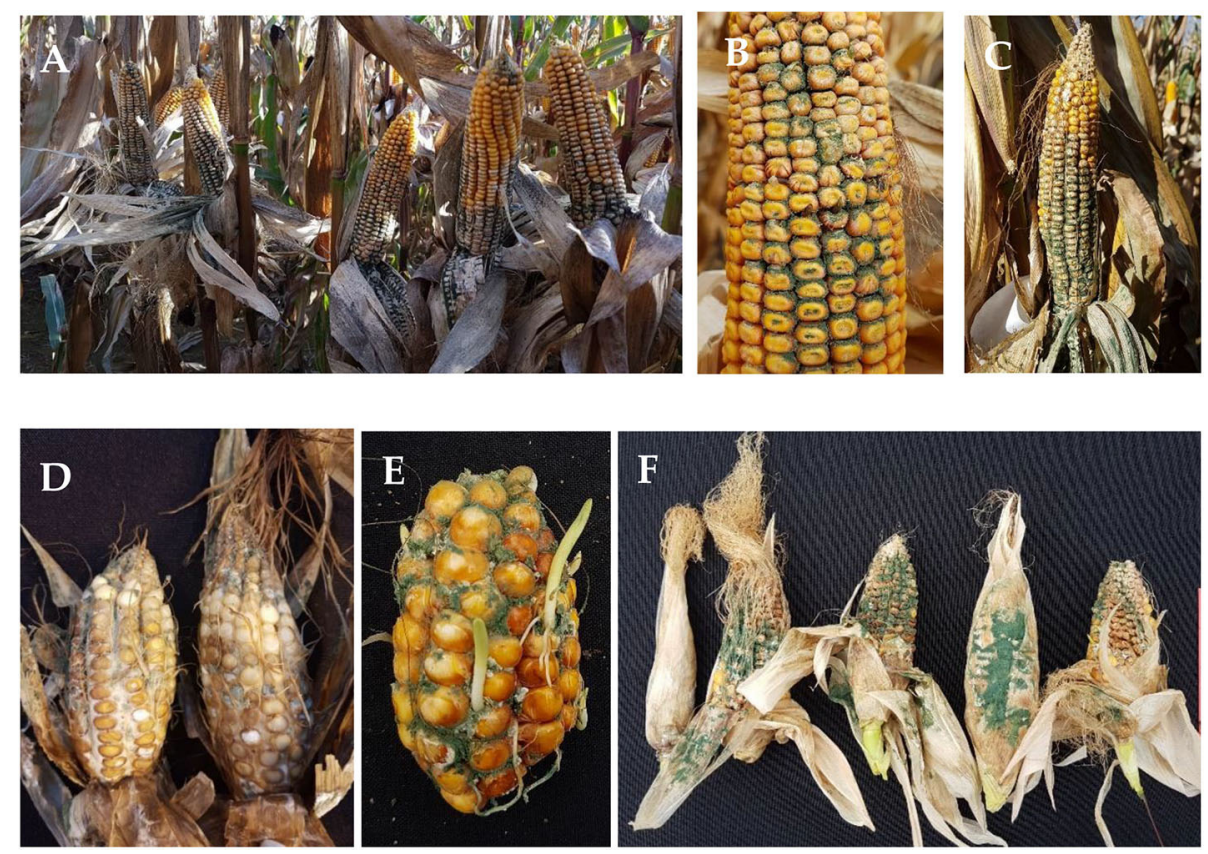

FIGURE 2 | Disease symptoms of natural infection with Trichoderma sp. on maize cobs in the field, (A) at Künzing, (B) at Bernburg, (C) and at Altoetting; (A-C); and after silk channel inoculation in the greenhouse (D-F).

characterized by white mycelium growing between the kernels and on the husk leaves with massive production of green to graygreen conidia. Under natural infection in the field, symptoms occurred from the base to the middle part of the cob, covering all kernels, and all layers of husk leaves (Figures 2A-C). No mechanical damage or injuries by birds or insects were observed on infected cobs. After inoculation of ears in the greenhouse, the whole cob, inside and outside of the husk leaves was covered with mycelium, and a green layer of conidia (Figures 2D-F). Some infected cobs in the field as well as in the greenhouse showed premature ripening of the kernels (Figures 2C,E).

After artificial inoculation of the cob in the greenhouse, six isolates were pathogenic on maize ears and ten isolates did not induce any infection. The highest disease severity was observed with $T$. afroharzianum isolates Tri5 (96.0\%), Tri1 (94.0\%), Tri2 (91.1\%), and Tri3 (78.0\%), which overgrew the kernels and husk leaves (Table 2). Isolates T12 and T39 (Trichodex) lead to greenish discoloration only at the tip of the cob. The reference strains of $T$. afroharzianum (CBS 124620), T.harzianum (IPP0320, IPP0318) and the two remaining strains (IPP0316 and Tri4) as well as the T. harzianum isolates from maize cobs (Tri6, Tri7, Tri8, Tri10, and Tri11) were non-pathogenic on maize. Nonpathogenic strains did not cause any significant reduction in dry matter content compared to water treated, non-infected control cobs. However, the three most aggressive strains Tri2, Tril, and Tri5 of T. afroharzianum caused significant losses in dry matter content of cobs 28 days after inoculation (Table 1).

\section{DISCUSSION}

Although Trichoderma is known as a plant symbiont or antagonist of fungal phytopathogens, our findings support previous observations in the US (Munkvold and White, 2016; Wise et al., 2016) that Trichoderma can infect maize cobs and cause ear rot diseases. Molecular-phylogenetic analysis of partial translation elongation factor- $1 \alpha(t e f-1 \alpha)$ and internal transcribes spacer (ITS) genes revealed pathogenic isolates as T. afroharzianum. To the best of our knowledge this is the first report on T. afroharzianum as an ear rot pathogen in Europe. The disease symptoms described in this study are in agreement with the observations by Wise et al. (2016) indicating that infection of Trichoderma appeared as white mold associated with massive production of green or gray-green conidia between the kernels and husk leaves, often involving the entire ear and causing premature ripening of the kernels. In contrast to the report by Munkvold and White (2016), T. afroharzianum infection, in the present study, did not require any previous damage on husk leaves and was not associated with injuries from birds or insects. Furthermore, artificial inoculation studies in the greenhouse confirmed that high disease severity occurred without any mechanical wounding after silk channel injection. Therefore, we conclude that Trichoderma ear rot infection is not a result of, or promoted by mechanical injuries. This new pathogen cannot be regarded as a wound or opportunistic pathogen, as it is clearly able to infect kernel and husk leaf tissue and cause disease without any previous damages. We therefore propose to use the name "Trichoderma ear rot on maize" for this disease. 
TABLE 2 | Disease severity (\%) and dry matter content (\%) of maize cobs, 28 days after inoculation.

\begin{tabular}{|c|c|c|}
\hline Isolate & Disease severity [\%] & Dry matter content [\%] \\
\hline \multicolumn{3}{|l|}{ Water } \\
\hline Control & $0.0 \pm 0.0^{a}$ & $47.9 \pm 4.6^{a}$ \\
\hline \multicolumn{3}{|l|}{ T. harzianum } \\
\hline IPP0320 & $0.0 \pm 0.0^{a}$ & $48.5 \pm 2.5^{a}$ \\
\hline Tri6 & $0.0 \pm 0.0^{a}$ & $46.5 \pm 13.5^{a}$ \\
\hline IPP0318 & $0.0 \pm 0.0^{a}$ & $46.2 \pm 6.7^{a}$ \\
\hline Tri7 & $0.0 \pm 0.0^{a}$ & $41.6 \pm 4.7^{a}$ \\
\hline T39 & $11.0 \pm 6.5^{b}$ & $40.4 \pm 12.4^{\mathrm{a}}$ \\
\hline Tri11 & $0.0 \pm 0.0^{a}$ & $39.1 \pm 12.9^{a}$ \\
\hline Tri8 & $0.0 \pm 0.0^{a}$ & $37.2 \pm 6.1^{a}$ \\
\hline Tri10 & $0.0 \pm 0.0^{a}$ & $36.7 \pm 7.9^{a}$ \\
\hline $\mathrm{T} 12$ & $14.0 \pm 10.8^{b}$ & $36.7 \pm 5.8^{\mathrm{a}}$ \\
\hline \multicolumn{3}{|l|}{ T. afroharzianum } \\
\hline CBS 124620 & $0.0 \pm 0.0^{a}$ & $39.7 \pm 6.0^{a}$ \\
\hline Tri1 & $94.0 \pm 5.4^{d}$ & $26.6 \pm 4.1^{b}$ \\
\hline Tri2 & $91.1 \pm 10.5^{d}$ & $20.9 \pm 7.7^{b}$ \\
\hline Tri3 & $78.0 \pm 22.8^{c}$ & $34.3 \pm 6.8^{\mathrm{ab}}$ \\
\hline Tri5 & $96.0 \pm 8.9^{d}$ & $20.3 \pm 6.0^{a}$ \\
\hline \multicolumn{3}{|l|}{ Others } \\
\hline IPP0316 (T. atroviride) & $0.0 \pm 0.0^{a}$ & $49.9 \pm 15.0^{\mathrm{a}}$ \\
\hline Tri4 (T. tomentosum) & $0.0 \pm 0.0^{a}$ & $45.7 \pm 13.2^{a}$ \\
\hline
\end{tabular}

Means are given with standard deviation. Letters $a, b, c, d$, ab indicate significant differences between the isolates $(\alpha \leq 0.05)$.

Highest disease severity and highest losses of dry matter content were observed after inoculation with three T. afroharzianum (Tri1, Tri2, Tri3, and Tri5) strains; however, the reference type strain of $T$. afroharzianum CBS 124620 did not induce any disease symptoms on maize but may have lost its pathogenicity during cultivation for over 15 years. Otherwise, this may indicate a phylogenetic separation on the species or subspecies level of pathogenic and non-pathogenic strains. Further research is required to clarify the genetic differences between these strains within the $T$. afroharzianum cluster. Surprisingly, a low-level pathogenicity of the two T. harzianum strains which were considered for use as biocontrol agents was recorded. Although these strains are usually applied to the soil for control of soilborne diseases, the pathogenicity found in our greenhouse experiment with cob inoculation cannot be ignored with regard to risk analysis in the registration of Trichoderma strains as biocontrol agents. The question whether beneficial Trichoderma strains could mutate into aggressive plant pathogens is difficult to determine at this stage and requires further research.

\section{REFERENCES}

Arora, D., Bridge, P., and Bhatnagar, D. (2003). Fungal Biotechnology in Agricultural, Food, and Environmental Applications. Boca Raton, FL: CRC Press.
Besides these findings, several epidemiological aspects about Trichoderma ear rot remain to be elucidated. Firstly, it is not known how the conidia of Trichoderma reach and infect the maize ears under natural conditions in the field, which sources of inoculum exist, and whether there are any alternative hosts. Secondly, there are no reports so far about the effect of weather conditions or agronomic practices possibly favoring infection with Trichoderma. The aggressive strains of T. afroharzianum were restricted to warmer regions with enhanced maize production in Southern Germany and along the Rhine valley. This is consistent with our previous observations indicating enhanced Trichoderma infection in the field in years with high mean temperatures and low precipitation such as in 2018 and 2019 in Germany. Furthermore, the potential production of mycotoxins by aggressive strains of Trichoderma awaits examination. Finally, field monitoring to explore the spread of the disease in Europe and yield loss analyses under field conditions are required to assess the economic significance of Trichoderma ear rot disease in maize production.

\section{DATA AVAILABILITY STATEMENT}

The raw data supporting the conclusions of this article will be made available by the authors, without undue reservation, to any qualified researcher.

\section{AUTHOR CONTRIBUTIONS}

AP: conceptualization, resources, and writing-original draft preparation. AP and SS: methodology, validation, investigation, data curation, and visualization. AT, PK, and SS: writing-review and editing. AT: supervision. AT and PK: project administration and funding acquisition. All authors have read and agreed to the published version of the manuscript.

\section{FUNDING}

This research was funded by the German Federal Office for Agriculture and Food (BLE) (Grant no. 2818208315).

\section{ACKNOWLEDGMENTS}

We thank Brigitte Jünemann and Chelsea Schreiber for their excellent technical assistance.

\section{SUPPLEMENTARY MATERIAL}

The Supplementary Material for this article can be found online at: https://www.frontiersin.org/articles/10.3389/fagro. 2020.547758/full\#supplementary-material

Brandfass, C., and Karlovsky, P. (2008). Upscaled CTAB-based DNA extraction and real-time PCR assays for Fusarium culmorum and F. graminearum DNA in plant material with reduced sampling error. Int. J. Mol. Sci. 9, 2306-2321. doi: 10.3390/ijms 9112306 
Chaverri, P., Branco-Rocha, F., Jaklitsch, W., Gazis, R., Degenkolb, T., and Samuels, G. J. (2015). Systematics of the Trichoderma harzianum species complex and the re-identification of commercial biocontrol strains. Mycologia 107, 558-590. doi: 10.3852/14-147

Clark, K., Karsch-Mizrachi, I., Lipman, D. J., Ostell, J., and Syaers, E. W. (2015). GenBank. Nucleic Acid Res. 44, D67-72. doi: 10.1093/nar/gkv1276

European and Mediterranean Plant Protection Organization. (2015). PP 1/285 (1) fusarium ear rot of maize. Bull. OEPP/EPPO Bull. 45, 336-339. doi: 10.1111/epp.12240

Ferrigo, D., Raiola, A., Piccolo, E., Scopel, C., and Causin, R. (2014). Trichoderma harzianum T22 induces in maize systemic resistance against Fusarium verticillioides. J. Plant Pathol. 96, 133-142. doi: 10.4454/JPP.V96I1.038

Ghazanfar, M. U., Raza, M., Raza, W., and Qama, M. I. (2018). Trichoderma as potential biocontrol agent, its exploitation in agriculture, a review. Plant Protect. 2, 2617-1279.

Gupta, V. K., Schmoll, M., Herrera-Estrella, A., Upadhyay, R. S., Druzhinina, I. S., and Tuohy, M. (2014). Biotechnology and Biology of Trichoderma. Amsterdam: Elsevier.

Harman, G. E. (2015). Overview of mechanisms and uses of Trichoderma spp. Phytopathology 96, 190-194. doi: 10.1094/PHYTO-96-0190

Harman, G. E., Howell, C. R., Viterbo, A., Chet, I., and Lorito, M. (2004a). Trichoderma species opportunistic, avirulent plant symbionts. Nat. Rev. Microbiol. 2, 43-56. doi: 10.1038/nrmicro797

Harman, G. E., Petzoldt, R., Comis, A., and Chen, J. (2004b). Interactions between Trichoderma harzianum strain T22 and maize inbred line Mo17 and effects of these interactions on diseases caused by Pythium ultimum and Colletotrichum graminicola. Biol. Control 94, 147-153. doi: 10.1094/PHYTO.2004.94. 2.147

Herrera-Estrella, A., and Chet, I. (2004). "The biological control agent Trichoderma from fundamentals to applications," in Handbook of Fungal Biotechnology, 2nd Edn, ed D. Aurora (Boca Raton, FL: CRC Press), 147-156. doi: 10.1201/9780203913369.ch13

Jaklitsch, W. M., and Voglmayr, H. (2015). Biodiversity of Trichoderma (Hypocreaceae) ins southern Europe and Macaronesia. Stud. Mycol. 80, 1-87. doi: 10.1016/j.simyco.2014.11.001

Kubicek, C. P., Komon-Zelazowska, M., and Druzhinina, I. S. (2008). Fungal genus Hypocrea/Trichoderma: from barcodes to biodiversity. J. Zhejiang Univ. 9, 753-763. doi: 10.1631/jzus.B0860015

Kumar, S., Stecher, G., and Tamura, K. (2016). MEGA7: molecular evolutionary genetics analysis version 7.0 for bigger datasets. Mol. Biol. Evol. 33, 1870-1874. doi: 10.1093/molbev/msw054

Munkvold, G. P., and White, D. G. (2016). Compendium of Corn Diseases, 4th Edn. St. Paul, MN: USA APS Press.

O'Donnell, K., Cigelnik, E., and Nirenberg, H. I. (1998). Molecular systematics and phytogeography of the Gibberella fujikuroi species complex. Mycologia 90, 465-493. doi: 10.1080/00275514.1998. 12026933

OSU. (2020). Iowa State University Trichoderma Ear Rot, Troubleshooting Abnormal Corn Ears. Available online at: https://u.osu.edu/mastercorn/ Trichoderma-ear-rot/ (accessed February 18, 2020).

Popiel, D. I., Koczyk, G., Dawidziuk, A., Gromadzka, K., Blaszczyk, L., and Chelkowski, J. (2014). Zearalenone lactonohydrolase activity in Hypocreales and its evolutionary relationships within the epoxide hydrolase subset of a/b-hydrolases. BMC Microbiol. 14:82. doi: 10.1186/1471$2180-14-82$

Samuels, G. J., and Hebbar, P. K. (2015). Trichoderma: Identification and Agricultural Applications. St. Paul, MN: American Phytopathological Society.

Schuster, A., and Schmoll, M. (2010). Biology and biotechnology of Trichoderma. Appl. Microbiol. Biotechnol. 87, 787-799. doi: 10.1007/s00253-0102632-1

Tamura, K., Stecher, G., Peterson, D., Filipski, A., and Kumar, S. (2013). MEGA6: molecular evolutionary genetics analysis version 6.0. Mol Biol Evol. 30, 2725-2729. doi: 10.1093/molbev/mst197

Thompson, J. D., Higgins, D. G., and Gibson, T. J. (1994). CLUSTAL W: Improving the sensitivity of progressive multiple sequence alignment through sequence weighting, position-specific gap penalties and weight matrix choice. Nucleic Acids Res. 22, 4673-4680.

Vincelli, P. (2014). Trichoderma Ear Rot of Corn. Kentucky Pest News. Available online at: https://kentuckypestnews.wordpress.com/2014/12/23/ Trichoderma-ear-rot-of-corn/ (accessed February 18, 2020).

White, T. J., Bruns, T., Lee, S., and Taylor, J. (1990). “Amplification and direct sequencing of fungal ribosomal RNA genes for phylogenetic," in PCR Protocols: A Guide to Methods and Applications, eds M. A. Innis, D. H. Gelfand, J. J. Snisky, and T. J. White (San Diego, CA: Academic Press Inc).

Wise, K., Allen, T., Chilvers, M., Faske, T., Freije, A., Isakeit, T., et al. (2016). Ear Rots. Crop Protection Network. Available online at: https://crop-protectionnetwork.s3.amazonaws.com/publications/cpn-2001-ear-rots.pdf (accessed March 10, 2020).

Conflict of Interest: The authors declare that the research was conducted in the absence of any commercial or financial relationships that could be construed as a potential conflict of interest.

Copyright (c) 2020 Pfordt, Schiwek, Karlovsky and von Tiedemann. This is an openaccess article distributed under the terms of the Creative Commons Attribution License (CC BY). The use, distribution or reproduction in other forums is permitted, provided the original author(s) and the copyright owner(s) are credited and that the original publication in this journal is cited, in accordance with accepted academic practice. No use, distribution or reproduction is permitted which does not comply with these terms. 